\title{
Vacinação do Cardiopata contra COVID-19: As Razões da Prioridade
}

\author{
Vaccinating Patients with Heart Disease Against COVID-19: The Reasons for Priority
}

\author{
Wolney de Andrade Martins, ${ }^{1,2,3}$ Gláucia Maria Moraes de Oliveira, ${ }^{4,5}$ Andréa Araujo Brandão, ${ }^{5,6}$ ๑ Ricardo \\ Mourilhe-Rocha, ${ }^{5,6,7}$ Evandro Tinoco Mesquita, ${ }^{1,3,5}$ José Francisco Kerr Saraiva, ${ }^{5,8}$ Fernando Bacal, ${ }^{5,9}$ Marcelo \\ Antônio Cartaxo Queiroga Lopes ${ }^{5}$ \\ Universidade Federal Fluminense (UFF), ${ }^{1}$ Niterói, RJ - Brasil \\ Sociedade de Cardiologia do Estado do Rio de Janeiro (SOCERJ), ${ }^{2}$ Rio de Janeiro, $R$ J - Brasil \\ Complexo Hospitalar de Niterói (CHN), ${ }^{3}$ Niterói, RJ - Brasil \\ Universidade Federal do Rio de Janeiro (UFRJ), ${ }^{4}$ Rio de Janeiro, RJ - Brasil \\ Sociedade Brasileira de Cardiologia (SBC), ${ }^{5}$ Rio de Janeiro, RJ - Brasil \\ Universidade do Estado do Rio de Janeiro (UERJ), ${ }^{6}$ Rio de Janeiro, RJ - Brasil \\ Hospital Pró-Cardíaco, Rio de Janeiro, ${ }^{7}$ RJ - Brasil \\ Pontifícia Universidade Católica de Campinas (PUC), ${ }^{8}$ Campinas, SP - Brasil \\ Universidade de São Paulo (USP), ${ }^{9}$ São Paulo - Brasil
}

\section{A pandemia da COVID-19}

A Organização Mundial da Saúde reconheceu a COVID-19 como pandemia em 11 de março de 2020 e, desde então, essa emergência de saúde pública converteu-se na principal causa de óbitos no mundo, o que tornou seu enfrentamento uma prioridade inquestionável. Ao escrevermos este editorial contabilizávamos 86.969.386 casos confirmados e 1.915.657 mortes por COVID-19 no mundo, dos quais 8.013.708 casos ocorreram no Brasil e resultaram em 201.460 mortes. ${ }^{1}$ Segundo as projeções do Institute for Health Metrics and Evaluation (IHME)² o Brasil atingirá 248.476 mortes por COVID-19 em 4 de abril de 2021. As projeções para o dia 19 de março de 2021 estimam 242.738 [232.202 - 255.044] mortes, que poderiam ser reduzidas para 241.668 [231.337 - 253.770], caso a vacina fosse rapidamente administrada, e para 223.910 [215.565 - 233.360], caso a máscara fosse utilizada em 95\% das situações em todos os locais. Preocupa a magnitude do número de casos e óbitos por única doença em tão pouco tempo. No momento, quando há crescimento de novos casos e internações, começar a vacinar terá impacto na redução de mortes e internações em intervalo curto. Apesar do esforço da comunidade científica, não há um tratamento específico para bloquear a replicação viral. Nesse sentido, programas de vacinação são poderosos aliados e, em virtude do notável progresso da ciência, já dispomos desse recurso.

O Brasil, por intermédio do Sistema Único de Saúde (SUS), tem se notabilizado pelo êxito da execução de programas de vacinação da sua população. É premente a instituição de política pública para vacinar dentro dos princípios do SUS: universalidade, integralidade e equidade. Entretanto, diante dos movimentos

\section{Palavras-chave}

Infecções por Coronavírus; COVID-19; Betacoronavírus; Pandemia; Vacinação; Vacinas; Doenças Cardiovasculares; Influenza Humana; Política de Saúde.

Correspondência: Wolney de Andrade Martins •

Universidade Federal Fluminense - Medicina Clínica - Rua Marques do

Paraná, 303, 6o Andar. CEP 24030215, Centro, Niterói, RJ - Brasil

E-mail: wolney_martins@hotmail.com

DOI: https://doi.org/10.36660/abc.20210012 antivacina que têm surgido em nível mundial, é necessário um forte esforço para que se obtenha a adesão da população. No passado, já enfrentamos com muito êxito essa incredulidade, como na Revolta da Vacina vivida por Oswaldo Cruz. Miremo-nos nesse exemplo para superar essa grave crise sanitária.

\section{A epidemiologia das doenças cardiovasculares na COVID-19}

No Brasil, entre 17 de março e 22 de maio de 2020, houve número maior de óbitos nas capitais das regiões Norte, Nordeste e Sudeste, especialmente em São Paulo, Rio de Janeiro, Fortaleza, Recife, Belém e Manaus, com menor ocorrência de notificação de óbitos nas capitais do Sul e do Centro-Oeste e nos municípios do interior. Observamos aumento de notificação de óbitos por causas cardiovasculares inespecíficas em todas as regiões, nas capitais e no interior, principalmente nas regiões Norte, Nordeste e Sudeste. Por outro lado, houve redução percentual das notificações de óbitos por síndrome coronariana aguda (SCA) e acidente vascular cerebral (AVC), com maior magnitude no Nordeste, seguindo-se as regiões Centro-Oeste e Sudeste (capital e interior). ${ }^{3}$

A pandemia pelo coronavírus em 2020 no Brasil aumentou o número de óbitos gerais, por doenças cardiovasculares (DCV) e por causas inespecíficas, assim como o número de mortes súbitas em domicílio. As diferenças regionais exprimem as desigualdades socioeconômicas e étnicas de um país continental, sendo ainda consequência de um sistema de saúde com recursos heterogêneos e mal distribuídos. ${ }^{3}$

COVID-19 é a novidade pandêmica. A DCV é nossa realidade endêmica, consolidada e irresoluta. Ambas comprometem a saúde em todos os aspectos, individuais e coletivos, físicos, psíquicos, sociais e econômicos. Em comum, ceifam vidas produtivas e promissoras.

Ainda carecemos de estudos duplo-cegos, randomizados, placebo-controlados que mostrem a relação de causalidade entre vacinação contra COVID-19 e benefício nos cardiopatas. Utilizemos então a melhor evidência disponível.

\section{As vacinas e o impacto na humanidade}

Apesar de terem surgido antes mesmo dos imunologistas, as vacinas provocaram impacto no controle ou até erradicação de doenças outrora devastadoras. A varíola matava 29\% das crianças 
na Londres dos séculos XVII e XVIII e foi declarada extinta em 1980. Quem entre nós fez diagnóstico de miocardite diftérica nos últimos 10 anos? Quantos casos de tétano neonatal foram internados em seu hospital em 2020? Vacinações mudaram a história natural das epidemias de difteria em 1940, de poliomielite em 1956, de coqueluche em 1950, de sarampo em 1968, de doença meningocócica em 1999, entre muitas outras. Entretanto, o vacilo nas campanhas resultou, invariavelmente, em reincidência. ${ }^{4}$

\section{O modelo 'influenza'}

A vacinação contra influenza é a experiência exitosa baseada em evidências mais próxima da atual situação pandêmica pela COVID-19. Apesar de a vacinação contra influenza ser recomendada pelas principais diretrizes em cardiologia, a cobertura vacinal é baixa e aumentou pouco na última década. ${ }^{5}$ A vacinação depende, em muito, da recomendação do cardiologista, que é, sobretudo, "o clínico" do cardiopata, ouvido em diversas situações. O conhecimento e consequente convencimento sobre a necessidade da vacina é determinante para sua difusão. A vacina da influenza é o exemplo inequívoco: achase disponível, é de fácil acesso em campanhas, mas sua cobertura não ultrapassa 25\% dos pacientes com insuficiência cardíaca (IC). 5,6
A necessidade da vacinação contra influenza em cardiopatas foi determinada primeiramente pelos relatos históricos de aumento de mortalidade nas epidemias e, posteriormente, por estudos epidemiológicos. ${ }^{5} \mathrm{O}$ Quadro 1 apresenta evidências que embasaram tais recomendações. ${ }^{7-15}$ Hoje, sabe-se que a vacinação é medida eficaz na prevenção secundária, pois reduz internações hospitalares por IC, AVC e SCA, além de reduzir mortalidade geral de modo mais expressivo que muitos medicamentos ou intervenções. ${ }^{5,6}$

\section{As infecções e a síndrome inflamatória sistêmica}

A influenza predispõe a pneumonia bacteriana secundária e, dessa forma, descompensa o paciente com IC. Sim, é fato. Entretanto, deve-se ressaltar que a síndrome inflamatória sistêmica secundária à influenza leva a alteração dos fatores de coagulação, hiperagregabilidade plaquetária, além de aumento das proteínas de fase inflamatória, das citocinas e do fator de necrose tumoral. Consequentemente, tem-se incremento dos fenômenos trombóticos e depósito de fibrina, hipocontratilidade do cardiomiócito, inflamação e aceleração da aterogênese e do remodelamento (Figura 1). Assim, explicase facilmente o porquê da redução de SCA e AVC nos pacientes

\section{Quadro 1 - Principais evidências que embasaram a recomendação da vacinação contra influenza em cardiopatas}

\begin{tabular}{|c|c|c|c|}
\hline Autor & Ano & $\mathbf{n}$ & Principais conclusões \\
\hline Nichol KL et al. ${ }^{7}$ & 2003 & 286.383 idosos & $\begin{array}{l}\text { Vacina contra influenza reduziu mortalidade geral em } 48 \% \text {, hospitalizações por doença cardíaca } \\
\text { em } 19 \% \text { e AVC entre } 16 \% \text { e } 23 \%\end{array}$ \\
\hline Yap FHY et al..$^{8}$ & 2004 & 17.226 internações por DCNT & Influenza causou excedente de $45,6 \%$ de internações por IC \\
\hline Sandoval $\mathrm{C}$ et al. ${ }^{9}$ & 2008 & $\begin{array}{l}5.448 \text { pacientes com } \\
\text { disfunção ventricular sistólica }\end{array}$ & $\begin{array}{l}\text { O risco de hospitalização por IC é } 8 \% \text { a } 10 \% \text { maior durante a estação de influenza, } \\
\text { independentemente de como é definida }\end{array}$ \\
\hline Jorge JEL et al. ${ }^{10}$ & 2009 & 6.596 hospitalizações por IC & $\begin{array}{l}\text { A sazonalidade com maior número de internações por IC descompensada ocorre também em } \\
\text { regiões de clima tropical }\end{array}$ \\
\hline $\begin{array}{l}\text { Estabragh ZR \& } \\
\text { Mamas } \text { MA }^{11}\end{array}$ & 2013 & 40 estudos & $\begin{array}{l}\text { Influenza leva a efeito direto: miocardite com choque cardiogênico, aumento de IAM, diminuição } \\
\text { da mortalidade cardiovascular após vacinação }\end{array}$ \\
\hline Wu WC et al. ${ }^{12}$ & 2014 & 107.045 pacientes com IC & Vacinação contra influenza reduziu mortalidade de pacientes com IC em 30 dias e 1 ano \\
\hline Caldeira D et al. ${ }^{13}$ & 2015 & 4 estudos & $\begin{array}{l}\text { Vacinação contra influenza é eficaz na prevenção secundária em pacientes com doença } \\
\text { cardiovascular. Faltam dados para comprovar a mesma ação em prevenção primária }\end{array}$ \\
\hline $\begin{array}{l}\text { Blaya-Nováková V } \\
\text { et al. }{ }^{14}\end{array}$ & 2016 & $\begin{array}{l}227.984 \text { pacientes seguidos } \\
\text { por } 5 \text { anos }\end{array}$ & Vacinação contra influenza reduziu risco de mortalidade global no inverno em $41 \%$ por ano \\
\hline Fang YA et al. ${ }^{15}$ & 2016 & $\begin{array}{l}4.406 \text { pacientes com IRC e } \\
\text { idade } \geq 55 \text { anos. }\end{array}$ & $\begin{array}{l}\text { Idosos com doença renal crônica que receberam vacinação anual contra influenza apresentam } \\
\text { menor risco de hospitalizações por IC }\end{array}$ \\
\hline
\end{tabular}

AVC: acidente vascular cerebral; DCNT: doenças crônicas não transmissiveis; IC: insuficiência cardiaca; IAM: infarto agudo do miocárdio; IRC: insuficiência renal crônica.

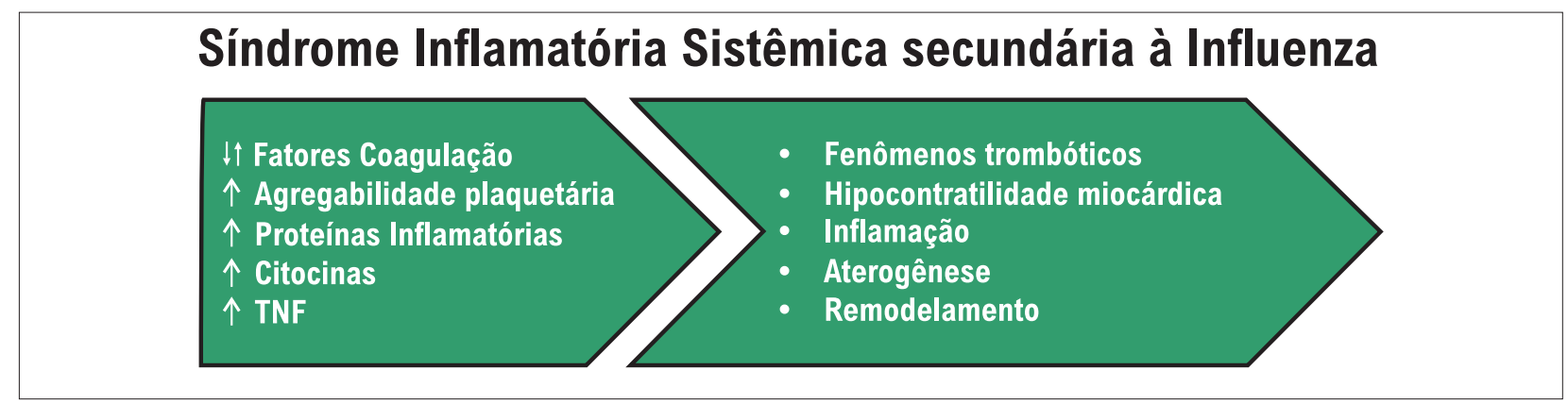

Figura 1 - Fisiopatologia das alterações cardiovasculares secundárias à inflamação sistêmica na influenza. 
vacinados em relação aos controles nos ensaios clínicos e observações epidemiológicas. ${ }^{5,16}$

A COVID-19 trouxe à tona a discussão dos mesmos mecanismos e manifestações já muito bem estudados na influenza. É inegável que a resposta inflamatória apresentada na COVID-19 seja mais exuberante e grave, associada ao risco de trombose. Portanto, conhecemos as peculiaridades da imunização nesse subgrupo de indivíduos e somos capazes de recomendar providências eficientes para ampliar as chances de sucesso do programa de imunização contra a COVID-19.

\section{COVID-19 e grupos de risco}

Desde as primeiras séries publicadas a partir da China e da Itália, a gravidade da COVID-19 sobressaiu nos portadores das doenças crônicas não transmissíveis, muito provavelmente tomados em comum pela inflamação sistêmica crônica. ${ }^{17}$ Descontadas as confusões suscitadas por interpretações inadequadas de estudos ecológicos, o conceito de grupo de risco manteve-se nas publicações subsequentes. Na verdade, fato já conhecido desde os estudos da influenza. O paciente com IC é um indubitável exemplo de grupo prioritário e a Sociedade Brasileira de Cardiologia (SBC) já se manifestou a respeito. ${ }^{18}$

Recentemente, a SBC foi convidada pelo Ministério da Saúde para integrar a Câmara Técnica e revisar o Programa Nacional de Imunização contra a COVID-19 e apontou sugestões relativas à vacinação em enfermos acometidos por todas as DCV, definindo e especificando grupos prioritários para a vacinação (Quadro 2).

\section{As atuais perspectivas com as diferentes vacinas contra COVID-19}

Ainda há poucas vacinas testadas em estudos fase 2 ou 3. No entanto, os resultados são muito positivos e impactantes, tanto em segurança quanto em eficácia. Merece destaque que as vacinas apoiadas pela Pfizer, ${ }^{19}$ Moderna $^{20}$

Quadro 2 - Doenças cardiovasculares e cerebrovasculares prioritárias para vacinação contra COVID-19. Sugestões oferecidas pela Sociedade Brasileira de Cardiologia ao Programa Nacional de Imunizações do Ministério da Saúde

\begin{tabular}{|c|c|}
\hline $\begin{array}{l}\text { Síndrome/Doença cardiovascular ou } \\
\text { cerebrovascular }\end{array}$ & Definição \\
\hline Insuficiência cardíaca & $\begin{array}{l}\text { - IC com fração de ejeção reduzida, intermediária ou preservada, em estágios B, C ou D, } \\
\text { independentemente de classe funcional da New York Heart Association } \\
\text { • Pós-transplante cardíaco (usar vacinas de vírus inativado) }\end{array}$ \\
\hline Cor-pulmonale e hipertensão pulmonar & Cor-pulmonale crônico, hipertensão pulmonar primária ou secundária \\
\hline Hipertensão arterial resistente & $\begin{array}{l}\text { Quando a PA permanece acima das metas recomendadas com o uso de três ou mais anti- } \\
\text { hipertensivos de diferentes classes, em doses máximas preconizadas e toleradas, administradas com } \\
\text { frequência, dosagem apropriada e comprovada adesão ou PA controlada em uso de quatro ou mais } \\
\text { fármacos anti-hipertensivos }\end{array}$ \\
\hline Hipertensão arterial estágio 3 & $\begin{array}{l}\text { PA sistólica } \geq 180 \mathrm{mmHg} \text { e/ou diastólica } \geq 110 \mathrm{mmHg} \text { independentemente da presença de LOA ou } \\
\text { comorbidade }\end{array}$ \\
\hline $\begin{array}{l}\text { Hipertensão arterial estágios } 1 \text { e } 2 \underline{\text { com LOA e/ }} \\
\text { ou comorbidade }\end{array}$ & $\begin{array}{l}\text { PA sistólica entre } 140 \text { e } 179 \text { mmHg e/ou diastólica entre } 90 \text { e } 109 \text { mmHg na presença de LOA e/ou } \\
\text { comorbidade }\end{array}$ \\
\hline Cardiopatia hipertensiva & $\begin{array}{l}\text { Cardiopatia hipertensiva (hipertrofia ventricular esquerda ou dilatação, sobrecarga atrial e ventricular, } \\
\text { disfunção diastólica e/ou sistólica, lesões em outros órgãos-alvo) }\end{array}$ \\
\hline Síndromes coronarianas & $\begin{array}{l}\text { Síndromes coronarianas crônicas (angina pectoris estável, cardiopatia isquêmica, pós infarto agudo } \\
\text { do miocárdio, outras) }\end{array}$ \\
\hline Valvopatias & $\begin{array}{l}\text { Lesões valvares com repercussão hemodinâmica ou sintomática ou com comprometimento miocárdico } \\
\text { (estenose ou insuficiência aórtica, estenose ou insuficiência mitral, estenose ou insuficiência pulmonar, } \\
\text { estenose ou insuficiência tricúspide, outras) }\end{array}$ \\
\hline Miocardiopatias e pericardiopatias & $\begin{array}{l}\text { Miocardiopatias de qualquer etiologia ou fenótipo } \\
\text { Pericardite crônica } \\
\text { Cardiopatia reumática }\end{array}$ \\
\hline $\begin{array}{l}\text { Doenças da aorta, dos grandes vasos e fístulas } \\
\text { arteriovenosas }\end{array}$ & Aneurismas, dissecções, hematomas da aorta e demais grandes vasos \\
\hline Arritmias cardíacas & $\begin{array}{l}\text { Arritmias cardíacas com importância clínica e/ou cardiopatia associada (fibrilação e flutter atriais, } \\
\text { outras) }\end{array}$ \\
\hline Cardiopatias congênitas no adulto & $\begin{array}{l}\text { Cardiopatias congênitas com repercussão hemodinâmica, crises hipoxêmica, insuficiência cardíaca, } \\
\text { arritmias, comprometimento miocárdico. }\end{array}$ \\
\hline $\begin{array}{l}\text { Próteses valvares e dispositivos cardíacos } \\
\text { implantados }\end{array}$ & $\begin{array}{l}\text { Portadores de próteses valvares biológicas ou mecânicas e dispositivos cardíacos implantados } \\
\text { (marca-passos, cardiodesfibriladores, ressincronizadores, assistência circulatória de média e longa } \\
\text { permanência) }\end{array}$ \\
\hline Doença cerebrovascular & Acidente vascular cerebral isquêmico ou hemorrágico, ataque isquêmico transitório, demência vascular \\
\hline
\end{tabular}

IC: insuficiência cardiaca; PA: pressão arterial; LOA: lesão de órgão-alvo. Fonte: correspondência enviada ao Programa Nacional de Imunizações do Ministério da Saúde, em 02/01/2021. 
e AstraZeneca ${ }^{21}$ incluíram idosos, cardiopatas, diabéticos, obesos graves, afrodescendentes e latinos. E, apesar do número relativamente reduzido, essa inclusão permite-nos inferir a segurança e a eficácia em pacientes cardiopatas. Os efeitos adversos observados foram locais, porém menos comuns nos mais idosos. Os efeitos cardiovasculares observados, como hipertensão, bradicardia, taquicardia, fibrilação atrial, SCA ou tromboembolia pulmonar, tiveram frequência menor que $0,1 \%$ e foram semelhantes entre os vacinados e os que receberam placebo (Quadro 3).

É oportuno destacar que o Brasil firmou parcerias, desde maio de 2020, para pesquisa e desenvolvimento de vacinas que incluem transferência de tecnologia por intermédio da Fundação Oswaldo Cruz e do Instituto Butantan. A vacina desenvolvida pela AstraZeneca e pela Universidade de Oxford já teve seus resultados preliminares publicados e encontra-se em uso na Inglaterra. Essa vacina será produzida em larga escala entre nós. Concomitantemente, a vacina denominada CoronaVac, desenvolvida pelo laboratório Sinovac, será produzida no Instituto Butantan. Esse Instituto informou nos meios de comunicação que "em estudo clínico com 12.400 voluntários, o imunizante demonstrou eficácia de 78\% para casos leves e de $100 \%$ para casos moderados e graves." 22 Portanto, há perspectivas objetivas de dispormos de vacinas.

É necessário enfatizar que o Brasil tem uma das mais avançadas legislações sanitárias do mundo. A Constituição Federal consagra o acesso à saúde como direito fundamental: "A saúde é um direito de todos e dever do Estado, garantido mediante políticas sociais e econômicas ...". Assim, políticas públicas de saúde seguras, eficazes, efetivas e custo-efetivas fazem parte do mínimo existencial de cada brasileiro, devendo ser ofertadas de maneira universal, integral e gratuita. Enquadram-se nesse contexto as campanhas de vacinação, verdadeiro patrimônio consolidado dos brasileiros e orgulho nacional. À vista disso, criar todas as condições para ofertar vacinas em um amplo programa de imunização contra a COVID-19 é "direito de todos e dever do Estado", sob pena do dever constitucional converter-se em promessa inconsequente, frustrando as justas expectativas depositadas no Estado brasileiro.

\section{Por que vacinar?}

Resumimos as dez razões para indicar a vacina ao seu paciente na Figura 2. É nosso ponto de vista, baseado na melhor

Quadro 3 - Características demográficas e clínicas dos voluntários vacinados contra COVID-19 nos ensaios clínicos

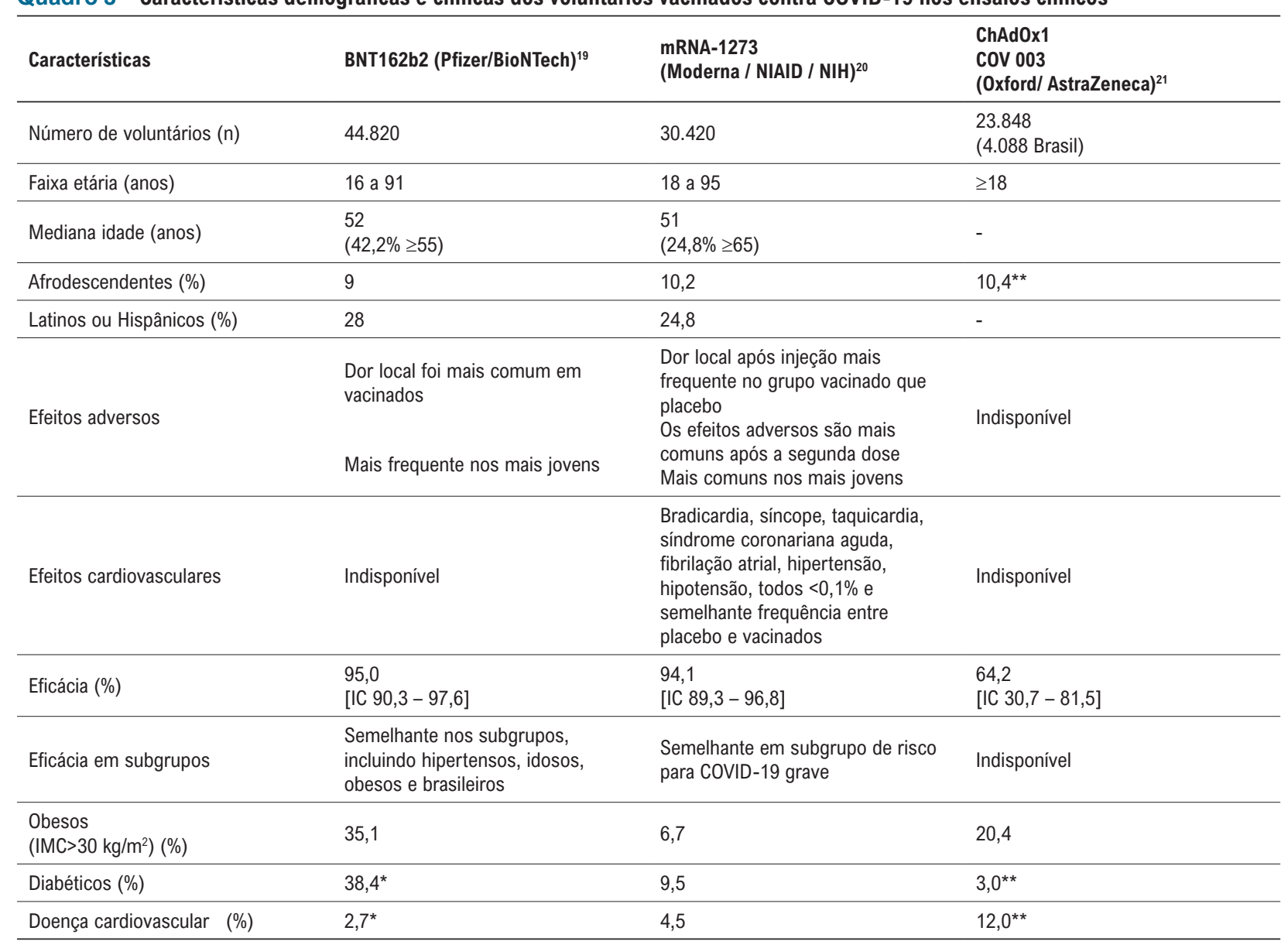

IMC: índice de massa corpórea. ( $\left.{ }^{*}\right)$ Cálculo aproximado a partir de dados publicados em apêndices do trabalho. (**) Dados referem-se à casuística do trabalho realizado no Brasil (COV 003). Nota: dados obtidos e analisados com critérios diferentes, portanto, com limitação quanto à comparação. 


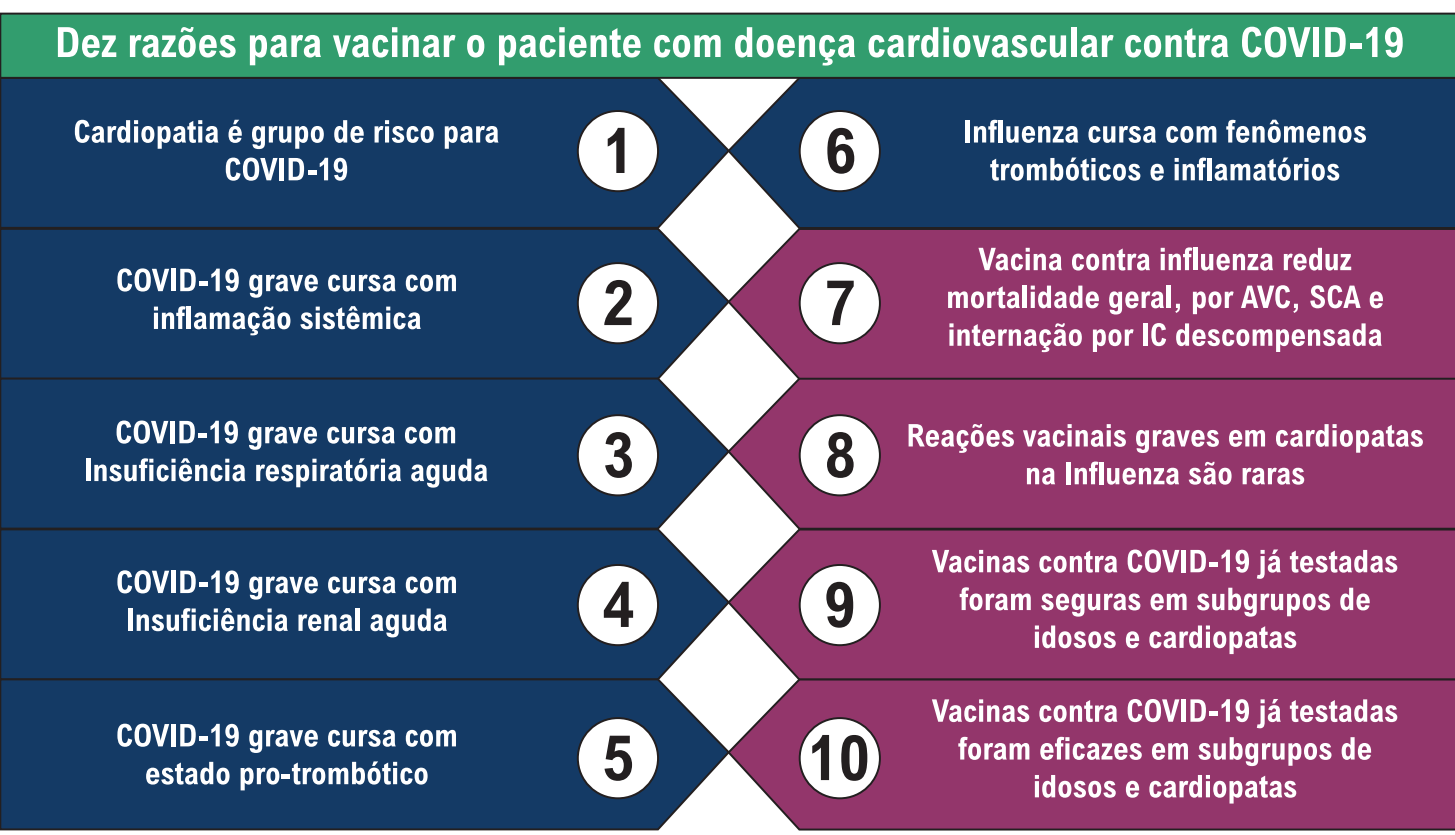

Figura 2 - Dez razões para vacinar o paciente com doença cardiovascular contra COVID-19. AVC: acidente vascular cerebral; SCA: síndrome coronariana aguda; IC: insuficiência cardiaca.

evidência existente, que devemos nos engajar na difusão desse conhecimento e motivar nossos pacientes. No entanto, impõese a manutenção das eficazes e comprovadas medidas de prevenção ao contágio pela COVID-19: higienização das mãos, uso de máscaras e distanciamento social. Em que pese que o programa de vacinação poderá contribuir para a minimização do contágio, certamente as medidas clássicas de prevenção deverão ser mantidas até que se prove definitivamente o benefício do programa de vacinação.

A Sociedade Brasileira de Cardiologia e seu compromisso com a ciência

A SBC não fugirá ao legado histórico, edificado no exemplo de Carlos Chagas, Dante Pazzanese e nossos pioneiros, transmitido por mais de sete décadas aos mais de 14.000 associados, confirmado em seu propósito social. É objetivo da SBC "Expandir, divulgar e incentivar, em todos os níveis, o conhecimento, o diagnóstico, a prevenção e o tratamento das DCV, desenvolvendo campanhas educativas em conjunto com o poder público e com outras entidades e associações, e divulgar, junto à sociedade civil, os aspectos epidemiológicos das DCV, esclarecendo-a quanto às possibilidades de prevenção e tratamento". ${ }^{23}$
Apesar do elevado custo em vidas perdidas, a busca por solução eficiente para a pandemia, trouxe-nos rápido avanço nas pesquisas, alicerçado em ciência de boa qualidade, deixando um notável legado e conquistas. No curso de um ano, descreveram-se o quadro clínico, o perfil epidemiológico e o agente etiológico em nível molecular, aprimoraram-se cuidados, refutaram-se tratamentos empíricos e fúteis e produziram-se vacinas testadas em ensaios clínicos. É a ciência em sua fascinante evolução por eficácia em prol da qualidade e quantidade de vida. Mas, a grande lição tem sido a necessidade do fortalecimento do sistema de saúde, o nosso SUS. A defesa intransigente do SUS, em síntese, é a defesa da dignidade da pessoa humana, compromisso fundamental do Estado brasileiro. A SBC e as demais sociedades científicas devem se aliar na luta pelo progresso e difusão da ciência e pela consecução de políticas públicas capazes de melhorar a vida de cada um dos mais de 220 milhões de brasileiros. Os princípios que nortearam a criação da SBC em 1943, no meio da Segunda Guerra Mundial, são os mesmos que nos motivam nesta crise sanitária sem precedentes. 


\section{Referências}

1. COVID-19 Dashboard by the Center for Systems Science and Engineering (CSSE) at Johns Hopkins University (JHU). Disponível em https:// coronavirus.jhu.edu/map.html. Acesso em 09/01/2021.

2. New IHME Projection Sees COVID-19 Deaths in Brazil at More than 125,000. Disponível em http://www.healthdata.org/news-release/newihme-projection-sees-covid-19-deaths-brazil-more-125000. Acesso em 06/01/2021.

3. Brant LCC, Nascimento BR, Teixeira RA, Lopes MACQ, Malta DC, Oliveira GMM, et al. Excess of cardiovascular deaths during the COVID-19 pandemic in Brazilian capital cities. Heart. 2020. Heart. 2020;106:1898-905. doi:10.1136/heartjnl-2020-317663.

4. Pollard AJ, Bijker EM. A guide to vaccinology: from basic principles to new developments. Nature Rev Immunol. 2020. Disponível em: https://doi. org/10.1038/s41577-020-00479-7. Acesso em 2020 em 05/01/2021.

5. Martins WA. Vacinação no cardiopata. In: Manual de Prevenção Cardiovascular/ [Editores Ricardo Mourilhe Rocha. Wolney Andrade Martins] Rio de Janeiro: SOCERJ, 2017. cap.8, p:87-93.

6. Martins WA, Ribeiro MD, Oliveira LB, Barros LS, Jorge AC, Santos CM, et al. Influenza and pneumococcal vaccination in heart failure: a little applied recommendation. Arq Bras Cardiol. 2011:96(3):240-5.

7. Nichol KL, Nordin J, Mullooly J, Lask R, Fillbrandt K, Iwane M. Influenza vaccination and reduction in hospitalizations for cardiac disease and stroke among the elderly. N Engl J Med.2003;348(14):1322-32.

8. Yap FHY, Ho PL, Lam KF, Chan PKS, Cheng YH, Peiris JSM. Excess hospital admissions for pneumonia, chronic obstructive pulmonary disease, and heart failure during influenza seasons in Hong Kong. J Med Virol. $2004 ; 73(4): 617-23$.

9. Sandoval C, Walter SD, Krueger P, Loeb MB. Comparing estimates of influenza-associated hospitalization and death among adults with congestive heart failure based on how influenza season is defined. BMJ Public Health. 2008;59(8). https://doi.org/10.1186/1471-2458-8-59

10. Jorge JEL, Cagyll M, Mesquita ET, Costa TLM, Moscavitch SD, Rosa MLG. Seasonal variation in hospitalizations due to heart failure in Niterói city, Southeastern Brazil. Rev Saúde Pública 2009;43(3):555-7.

11. Estabragh ZR, Mamas MA. The cardiovascular manifestations of influenza:a systematic review. Intern J Cardiol. 2013;167(6):2397-403.
12. Wu WC, Jiang L, Friedmann PD, Trivedi A. Association between process quality measures for heart failure and mortality among US veterans. Am Heart J 2014;168(5):713-20.

13. Caldeira $D$, Costa J, Vaz-Carneiro A. Vacina contra influenza para prevenção de doença cardiovascular. Acta Med Port. 2015;28(4):424-6.

14. Blaya-Nováková V, Prado-Galbarro FJ, Sarría-Santamera A. Effects of annual influenza vaccination on mortality in patients with heart failure. Eur J Public Health. 2016;26(5):890-2.

15. Fang YA, Chen Cl, Liu JC, Sung LC. Influenza vaccination reduces hospitalization for heart failure in elderly patients with chronic kidney disease:a populationbased cohort study. Acta Cardiol Sin. 2016;32(3):290-8.

16. BhattAS, DeVore AD, HernandezAF, Mentz RJ. Can vaccinations improve heart failure outcomes? JACC Heart Fail. 2017;5(3):194-203.

17. Wang D, Hu B, Hu C, Zhu F, Liu X, Zhang J, et al. Clinical characteristics of 138 hospitalized patients with 2019 novel coronavirus-infected pneumonia in Wuhan, China. JAMA. 2020;323;(11):1061-9.

18. Sociedade Brasileira de Cardiologia. Departamento de Insuficiência Cardíaca. A IC e a vacina no contexto da sindemia coronavírus 2020-21. Disponível em http://departamentos.cardiol.br/sbc-deic/profissional/noticias/20201228-aic-e-a-vacina-no-contexto-da-sindemia-coronavirus-2020-21.asp. Acesso em 09/01/2021.

19. Polack FP, Thomas SJ, Kitchin N, Absalon J, Gurtman A, Lockhart S, et al. Safety and efficacy of the BNT162b2 mRNA Covid-19 vaccine. N Engl J Med. 2020.383(27):2603-15. doi: 10.1056/NEJMoa2034577.

20. Baden LR, El Sahly HM, Essink B, Kotloff K, Frey S, Novak R, et al. Efficacy and safety of the mRNA-1273 SARS-CoV-2 vaccine. N Engl J Med. 2020 Dec 30. doi:10.1056/NEJMoa2035389.

21. Voysey M, Clemens SAC, Madhi SA, Weckx LY, Folegatti PM, Aley PK, et al. Safety and efficacy of the ChAdOx1 nCoV-19 vaccine (AZD1222) against SARS-CoV-2: an interim analysis of four randomised controlled trials in Brazil, South Africa, and the UK. Lancet. 2020. Jan 09;397(10269):99-111.https:// doi.org/10.1016/S0140-6736(20)32661-1.

22. Barifouse R. BBC News Brasil. CoronaVac: Butantan afirma que vacina evitou $78 \%$ de casos leves e 100\% de graves em testes. Disponível em https://www. bbc.com/portuguese/brasil-55262727. Acesso em 08/01/2021.

23. Sociedade Brasileira de Cardiologia. Estatuto Social. Disponível em http:// socios.cardiol.br/estatuto/EstatutoSBC_set2019.pdf. Acesso em 02/01/2021. 